\title{
COVID-19 Pandemic and Consecutive Changes in Geriatric Rehabilitation Structures and Processes - A Deeper Attempt to Explain the COVID Rehabilitation Paradox (Lessons to Learn to Ensure High Quality of Care in GR Services)
}

\author{
S. Grund ${ }^{1, *}$, A.L. Gordon', J.M. Bauer ${ }^{l}$, W.P.Achterberg ${ }^{3}$, J.M.G.A.Schols ${ }^{4,5}$
}

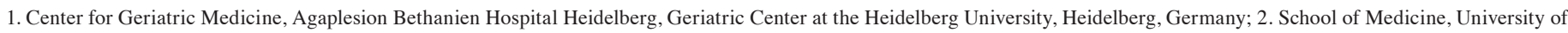

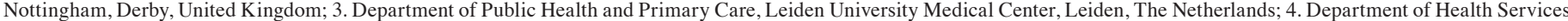

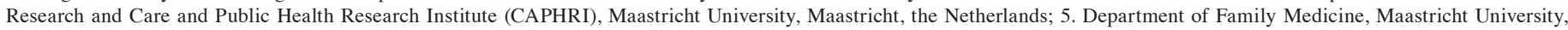
Maastricht, the Netherlands

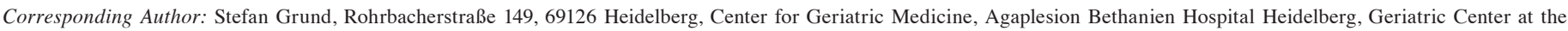
Heidelberg University, Heidelberg, Germany; stefan.grund@bethanien-heidelberg.de

\section{Introduction}

$\mathrm{O}$ lder people have been particularly adversely affected by the COVID-19 pandemic. By comparison with younger people, they have experience higher rates of hospitalisation and intensive care unit admissions (1-3), have had worse clinical outcomes $(4,5)$ and have required longer to recover $(6,7)$.

Geriatric rehabilitation (GR) has a particular role to play in the assessment and management of older people with multiple long-term conditions, frailty, disability and/or cognitive impairment (8). Because of the very high infection rates in these cohorts during second COVID-19 wave it is anticipated that the demand for GR will increase. Despite this, it has been suggested that the capacity for GR has reduced across Europe, as services have restructured to combat the pandemic. We have previously described this as the COVID-Rehabilitation Paradox (9).

To describe this situation in detail, we incorporated questions on COVID-19 into the "European Geriatric Medicine Society survey on best practice in geriatric rehabilitation». This survey was created to provide the basis for a European best practice guideline for geriatric rehabilitation.

\section{Methods}

An online survey was conducted to assess general best practice and pandemic-related changes in structures and processes of geriatric rehabilitation facilities across participating European countries.

The survey contained 3 sections with a total of 81 items. The first part of the survey asked about the general impact of the pandemic on GR services: such as capacity constraints, transfer or admission issues affecting GR, infection control mechanisms, and changes to working hours. The second part asked about GR service structures, processes and assessment domains, and the impact of the COVID-19 pandemic on each of these. It included questions on: rehabilitation team structure; systematic evaluation of GR services, use of guidelines and standards; rehabilitation process tools (e.g. CGA, treatment protocol); standard rehabilitation procedures (e.g. daily rounds, communication with family members or other health care providers); treatment duration; treatment frequency; and assessment domains. In the third part, assessments employed routinely and the influence of the pandemic was probed.

The survey was created with Google Forms and sent to the participants (EuGMS Geriatric Rehabilitation Working Group members) in April 2020. The members were from different European countries. Where respondents' responses were unclear, clarification was sought by follow-up email.

\section{Results}

\section{Data collection}

18 members of the EuGMS SIG-on GR from 8 European countries (BE, CH, FR, GER, IT, NL, SP, UK) completed the questionnaire. $44.4 \%(\mathrm{~N}=8)$ of the respondents were geriatricians, $16,7 \%(\mathrm{~N}=3)$ physicians, $22.2 \%(\mathrm{~N}=4)$ physiotherapists and $5.6 \%(\mathrm{~N}=1)$ occupational therapists, nurse practitioner or dieticians.

General influences of COVID-19 pandemic on GR services

\section{Capacity shortages}

$56 \%$ of the respondents stated that there were problems regarding capacity in geriatric rehabilitation. Reasons were: «Beds were blocked for possible COVID patients»: «COVID infections in GR patients affected patient flow»; «Fewer patients can be admitted due to environmental adaptations (e.g. empty beds due to isolation procedures». 


\section{Delayed admissions to GR}

$39 \%$ reported consistent delays due to pandemic situation and $17 \%$ could not determine whether existing delays were pandemic related or not. $44 \%$ of respondents reported that there no delays in admission/transfer to Geriatric Rehabilitation due to pandemic situation. Delays to admission to GR could be up to 15 days, as, patients were required to complete quarantine in other settings prior to transfer. Another participant reported that delays occurred because of capacity issues - keeping beds empty to maintain social distancing reduced capacity and hence patient flow.

One participant reported that admissions happened earlier and more rapidly than usual, because acute care departments were focused on making space for new patients with COVID19.

The implementation of cohorting or zoning guidance and effects of PPE on daily work are described in the Appendix 1 and 2 in the supplementary data on the journal website.

\section{Routine in GR service structures and changes due to COVID-19}

Nearly all professional groups were less involved in the treatment of patients as a consequence of COVID19. Respondents indicated access to Speech and Language Therapists (SALTs), social workers, and pharmacists to be most restricted, whilst geriatricians, nurses, physiotherapists and occupational therapists were more consistently available (Please see also Appendix 3 in the supplementary data on the journal website).

\section{Routine GR service processes and changes due to pandemic}

Almost all service processes were adapted due to the pandemic situation (Figure 1). The most significant changes were in daily rounds, transitional care (discharge management), outpatient GR after inpatient GR, and outcome measurement after discharge.

All routine process components were impacted by the pandemic. Caregiver training, regular team meetings, structured discharge planning, and comprehensive geriatric assessment were most affected (Figure 2). For changes in GR routine assessments and barriers to provide them due to COVID-19 pandemic please see also Appendix 4 and 5 in the supplementary data on the journal website.

\section{Discussion}

The main finding from this study is that COVID-19 resulted in substantial changes to the way that rehabilitation for older people was organised across eight European countries, with reduced capacity, reduced time spent per patient, and reduced access to members of the multidisciplinary team. Many of these changes took place as a consequence of infection control measures which restricted bed utilisation and complicated the workflow of rehabilitation staff. These data support the reduced capacity component of the rehabilitation paradox (9).

Figure 1. Routine GR service processes and frequency of impairment due to COVID-19 pandemic situation $(\mathrm{N}=18)$

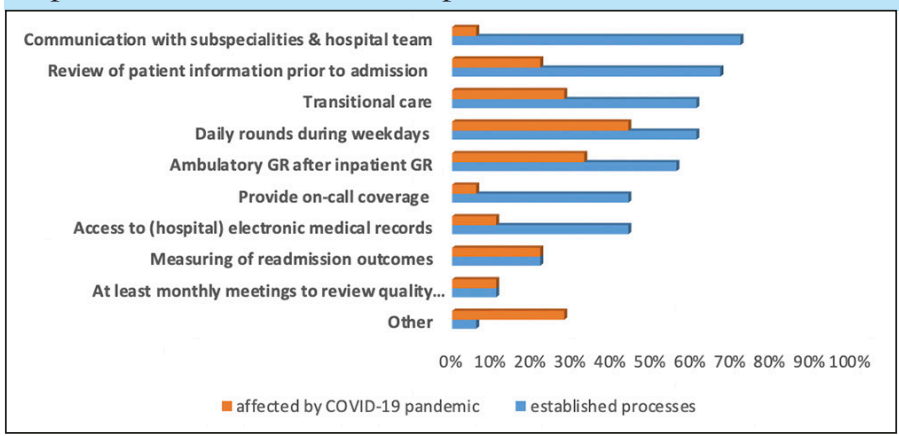

Figure 2. Routine GR processs tools and frequency of impairment due to COVID-19 pandemic $(\mathrm{N}=17)$

\begin{tabular}{|r} 
Regular multidisciplinary team meetings \\
Shared decision making \\
Treatment goals \\
Treatment plan
\end{tabular}

There were differences in the way in which the pandemic influenced care. Not all respondents had plans in place for cohorting and zoning, which is a cornerstone of health and safety recommendations for management of COVID-19 (10). Some respondents reported dramatic alterations to their working life, whilst others found the measures undertaken to prepare for COVID-19 less intrusive. This may reflect, to an extent, the differences in how GR is organized across Europe (11), with those services which are more fully staffed and better equipped being better able to absorb the additional workload associated with pandemic preparedness. The impact of the pandemic on service delivery across the continent does, however, speak to the need to plan proactively for future pandemics, epidemics or disasters that affect older people. Work undertaken to develop guidelines for long-term care during the pandemic (12), could easily be replicated for GR facilities, and designed in such a way that they could be applied to contexts other than COVID19.

Perhaps more important than preparedness of GR facilities for COVID-19, is the recognition that GR does not seem to have been widely recognized as part of the response to the pandemic. Across the continent, respondents described changes to admission criteria, or bed utilization, that seemed to consider GR facilities more as a flexible bed base, than as services that could play an active role in the pandemic response. The failure to increase GR capacity to take account of increased demand, with consequent bottlenecks and delays in intervention, shows a general lack of insight into the role the GR can play in enabling patients to recover after infection. Work underway by the 
EUGMS to raise the profile of GR more generally (8) will be important in helping policymakers and service leaders to build sufficient GR capacity into future pandemic preparedness.

The impact of altered team structures, and service processes, on rehabilitation outcomes is not yet clear, although it is unlikely to be positive. Ongoing studies, such as the COGER (13) and HERO study (14) will shed more light on this area and may help to further inform future planning.

The small number of participants may be a shortcoming of this work. But we recruited the participants as national representatives and leaders, with an overview of how care was proceeding within member countries. In addition, services have been subject, in most countries, to multiple iterative reconfigurations during the COVID-19 pandemic, so it is possible that a snapshot survey may only paint a small part of the picture. But with regard to the capacity changes during the first year of pandemic the first nationwide survey on structure changes in Germany support the rehabilitation paradox in inpatient geriatric rehabilitative care settings (15).

\section{Conclusion}

This study reports important changes in the structure and processes of GR across multiple European countries. It provides a sense that GR was not prioritised, or even protected, as part of the COVID-19 response. It is likely that services were rendered less effective, and that patients experienced worse outcomes, as a consequence. More detail will be forthcoming with the outcomes of important pending cohort studies about GR across Europe. It is certain, however, that to prevent similar deficiencies during future crises - including pandemics, epidemics, and natural and man-made disasters - that GR services need to be considered more fully as part of contingency planning.

Availability of data and materials: Data are available on request.

Conflicts of interest: The authors have no conflicts of interest to declare.

Funding: No funding.

Authors' contributions: The questionnaire was developed by Stefan Grund and Jos Schols and reviewed / revised by Adam Gordon, Wilco Achterberg and Jürgen Bauer. The first draft of the manuscript was written by Stefan Grund, which was reviewed and revised by J.M.G.A. Schols, A.L. Gordon, Jürgen Bauer and W.P Achterberg. Subsequently all authors commented on the revised version of the manuscript, which resulted in the final manuscript. The submission of the manuscript to this journal is approved by all authors
Ethics approval: Not applicable

Acknowledgements: The authors would like to thank all participants of the survey, the SIG-on GR members and the EuGMS who/which support this work for a better understanding of the changes in geriatric rehabilitation due to pandemics with the aim to improve the rehabilitative care of older people.

\section{References}

1. Cumulative rate of laboratory-confirmed COVID-19-associated hospitalizations in the United States as of September 26, 2020, by age group. Statista. (2020). https:// www.statista.com/statistics/1122354/covid-19-us-hospital-rate-byage/ (Accessed 10 September 2021)

2. Phua J, Weng L, Ling L et al. Intensive care management of coronavirus disease 2019 (COVID-19): challenges and recommendations. Lancet Respir Med 2020; 8: 506-17. https://doi.org/10.1016/S2213-2600(20)30161-2

3. Percentage of COVID-19 cases in the United States from February 12 to March 16 , 2020 that required intensive care unit (ICU) admission, by age group_. Stat (2020). https://www.statista.com/statistics/1105420/covid-icu-admission-rates-us-by-agegroup/ (Accessed 10 September 2021)

4. Number of coronavirus disease 2019 (COVID-19) deaths in the U.S. as of September 8, 2021, by age*. Statista (2021). https://www.statista.com/statistics/1191568/reporteddeaths-from-covid-by-age-us/ (Accessed 10 September 2021).

5 Number of coronavirus (COVID-19) deaths in Germany in 2021, by gender and age. Statista (2021). https://www.statista.com/statistics/1105512/coronavirus-covid-19. deaths-by-gender-germany/ (Accessed 10 September 2021).

6. Rees EM, Nightingale ES, Jafari Y, et al. COVID-19 length of hospital stay: a systematic review and data synthesis. BMC Med. 2020;18(1):270. https://doi. org/10.1186/s12916-020-01726-3

7. Medetalibeyoglu A, Senkal N, Kose M, et al. Older Adults Hospitalized with COVID19: Clinical Characteristics and Early Outcomes from a Single Center in Istanbul, Turkey. J Nutr Health Aging 2020; 24, 928-937 (2020). https://doi.org/10.1007/ s12603-020-1499-9

8. Grund S, Gordon AL, van Balen R, et al. European consensus on core principles and future priorities for geriatric rehabilitation: consensus statement. Eur Geriatr Med 2020;11(2):233-238. https://doi.org/10.1007/s41999-019-00274-1

9. Grund S, Gordon AL, Bauer JM, et al. The COVID Rehabilitation Paradox: why we need to protect and develop Geriatric Rehabilitation Services in the face of the pandemic. Age Ageing. 2021;50(3):605-607. https://doi.org/10.1093/ageing/afab009

10. Making your workplace COVID-secure during the coronavirus pandemic. Health and Safety Executive (HSE). https://www.hse.gov.uk/coronavirus/working-safely/index htm (Accessed 2. June 2021).

11. Grund S, van Wijngaarden JP, Gordon AL, et al. EuGMS survey on structures of geriatric rehabilitation across Europe. Eur Geriatr Med 2020; 11, 217-232. https://doi org/10.1007/s41999-019-00273-2

12. Blain H, Rolland Y, Schols JMGA, et al. August 2020 Interim EuGMS guidance to prepare European Long-Term Care Facilities for COVID-19. Eur Geriatr Med. 2020;11(6):899-913. https://doi.org/10.1007/s41999-020-00405-z

13. European Cooperation in geriatric rehabilitation research after COVID19 (EU-COGER) (2020) https://www.lumc.nl/org/unc-zh/English/Research/ GeriatricRehabilitation/EU_COGER_ENGLISHpage/; (Accessed 2 June 2021)

14. Home-based Extended Rehabilitation for Older people (HERO) (2017) https://njladmin.nihr.ac.uk/document/download/2025073 (Accessed 2 June 2021)

15. Grund S, Jamour M, Becker C, et al. Inpatient geriatrics: The COVID Care Paradox. Dtsch Arztebl 2021;118 (21): A 1044-9. https://www.aerzteblatt.de/archiv/219250/ Stationaere-Geriatrie-Das-COVID-Versorgungsparadox

How to cite this article: S. Grund, A.L. Gordon, J.M. Bauer, et al. COVID-19 Pandemic and Consecutive Changes in Geriatric Rehabilitation Structures and Processes - A Deeper Attempt to Explain the COVID Rehabilitation Paradox (Lessons to Learn to Ensure High Quality of Care in GR Services). J Nutr Health Aging. 2022;26(1):64-66, http://dx.doi.org/10.1007/s12603-021-1716-1 